\title{
Gentian violet inhibits MDA-MB-231 human breast cancer cell proliferation, and reverses the stimulation of osteoclastogenesis and suppression of osteoblast activity induced by cancer cells
}

\author{
MASAYOSHI YAMAGUCHI $^{1 *}$, TATYANA VIKULINA ${ }^{2}$ and M. NEALE WEITZMANN ${ }^{2,3 *}$ \\ ${ }^{1}$ Department of Hematology and Medical Oncology, Winship Cancer Institute, and ${ }^{2}$ Division of Endocrinology and \\ Metabolism and Lipids, Department of Medicine, Emory University School of Medicine, Atlanta, GA 30322, USA; \\ ${ }^{3}$ Atlanta Department of Veterans Affairs Medical Center, Decatur, GA 30033, USA
}

Received April 22, 2015; Accepted May 25, 2015

DOI: 10.3892/or.2015.4190

\begin{abstract}
Gentian violet (GV) is a cationic triphenylmethane dye, with potent antifungal and antibacterial activity. We recently reported that in vitro GV suppresses the differentiation of bone resorbing osteoclasts while stimulating the differentiation and activity of bone forming osteoblasts. Breast cancer is highly metastatic to bone and drives bone turnover that further promotes cancer engraftment and expansion, the so-called vicious cycle. In humans, breast cancer metastases cause osteolytic lesions and skeletal damage that leads to bone fractures, an additional source of patient morbidity. The MDA-MB-231 human breast cancer cell line is a commonly used model of human breast cancer that when injected into mice metastasizes to bone causing osteolytic lesions by promoting osteoclastic bone resorption and/or suppressing osteoblastic bone formation. In the present study, we investigated the direct action of GV on MDA-MB-231 proliferation, and the capacity of GV to reverse the negative impact of MDA-MB-231 cells on osteoclast and osteoblast differentiation. Our data reveal for the first time that GV suppresses proliferation, and induces apoptosis, of MDA-MB-231 cells. We further demonstrated the capacity
\end{abstract}

Correspondence to: Dr Masayoshi Yamaguchi, Department of Hematology and Medical Oncology, Winship Cancer Institute, Emory University School of Medicine, 1365 C. Clifton Road, NE, Atlanta, GA 30322, USA

E-mail: yamamasa1155@yahoo.co.jp

Dr M. Neale Weitzmann, Division of Endocrinology and Metabolism and Lipids, Department of Medicine, Emory University School of Medicine, 101 Woodruff Circle, 1329 WMRB, Atlanta, GA 30322, USA

E-mail: mweitzm@emory.edu

*Contributed equally

Key words: gentian violet, human breast cancer, MDA-MB-231, human pancreatic cancer, MIA PaCa-2, cell proliferation, apoptosis, mineralization, osteoclast, osteoblast, bone marrow of GV to reverse the pro-osteoclastogenic and anti-osteoblastic activities of MDA-MB-231 cells in vitro. These data suggest that GV has important applications in the treatment of breast cancer through multiple actions including direct suppression of cancer cell proliferation, breaking the vicious cycle between cancer and bone, and alleviating the skeletal defects induced by bone metastasis.

\section{Introduction}

Bone homeostasis is maintained by the coordinated action of osteoclasts, osteoblasts and osteocytes in bone tissues (1). Bone loss occurs when the rate of osteoclastic bone resorption exceeds that of osteoblastic bone formation $(2,3)$. The consequence of bone loss is the development of osteoporosis, a condition that significantly increases the propensity for bone fractures, a major public health threat. Numerous pathologic conditions promote bone loss including estrogen deficiency, inflammation, bacterial and viral infection, obesity and diabetes. Notably, several types of cancer, including $\mathrm{T}$ cell lymphoma, multiple myeloma, prostate and breast cancer have a propensity to metastasize to bone. Bone metastasis occurs in 70-80\% of patients with advanced breast cancer (4-7), leading to severe pathological bone fractures, intractable pain, hypercalcemia and spinal cord and nerve-compression syndromes $(6,8)$, that add significantly to patient morbidity and increase mortality.

Tumor invasion into bone tissues often promotes osteoclastic bone resorption, resulting in the liberation of growth factors from the bone matrix, which feed back on cancer cells to enhance tumor growth resulting in the so-called 'vicious cycle of bone metastasis' $(7,8)$. Breast cancer cells promote the formation of osteoclasts through secretion of osteoporotic cytokines, such as parathyroid hormone-related peptide (PTH-rP), prostaglandin $\mathrm{E}_{2}\left(\mathrm{PGE}_{2}\right)$, tumor necrosis factor- $\alpha$ (TNF- $\alpha$ ), interleukins (IL-1, IL-6, IL-8, IL-11, IL-15 and IL-17) and leukemia inhibitory factor (LIF) $(7,9)$. These factors ultimately converge on RANKL pathway to drive osteoclast formation and activity leading to bone resorption and osteolytic lesions.

Constitutively activated nuclear factor- $\kappa \mathrm{B}(\mathrm{NF}-\kappa \mathrm{B})$ in breast cancer cells has been shown to play a crucial role in 
the osteolytic mechanism of bone metastasis of breast cancer by stimulating production of granulocyte macrophagecolony stimulating factor (GM-CSF) in breast cancer cells that enhance osteoclast development from monocytes (10). Progesterone receptor-positive mammary epithelial cancer cells express receptor activator of NF- $\mathrm{B}$ ligand (RANKL) that mediates epithelial proliferation and carcinogenesis (11). Differentiation and activation of osteoclasts are stimulated by production of RANKL, which is stimulated by several osteoclastogenic cytokines including PTH-rP, TNF- $\alpha$ and interleukins in osteoblasts (12), and other cells. Furthermore, breast cancer causes an increase in osteoblast apoptosis and a decrease in osteoblastic products required for new bone formation (9). Thus, breast cancer bone metastasis-induced bone loss is due to both activated osteoclastic bone resorption and suppressed osteoblastic bone formation (13).

Therapeutic strategies to break the vicious cycle have centered on bisphosphonates or anti-RANKL antibody (denosumab) as the current standard of care for patients with bone metastasis (13). Bisphosphonates inhibit osteoclastic bone resorption by targeting active osteoclasts while denosumab diminishes the differentiation, maturation and activity of osteoclasts by inhibiting the association of RANKL with its receptor RANK on preosteoclasts and mature osteoclasts. These agents are only partly effective in alleviating breast cancer-induced skeletal damage and may themselves be associated with significant side-effects including further suppression of bone formation, osteonecrosis of the jaw and atypical femoral fractures.

Gentian violet $(\mathrm{GV})$, a triaminophenylmethane dye, has been used extensively in medicine for over a century owing to its potent anti-microbial action (14). Recent studies further suggest angiogenic and anticancer properties of $\mathrm{GV}$ and there is now renewed interest in the medical applications of this dye $(15,16)$. In a recent study, we found that GV exhibits anti-NF- $\kappa \mathrm{B}$ activity and similar to other $\mathrm{NF}-\kappa \mathrm{B}$ antagonists can potently augment osteoblast differentiation and mineralization, yet potently suppresses osteoclast differentiation (17).

In the present study, we utilized the MDA-MB-231 breast cancer cell model that has previously been shown by us to stimulate osteoclast formation and suppress osteoblast differentiation in vitro (18) to investigate the hypothesis that GV may break the vicious cycle between cancer cells and bone turnover, by diminishing cancer-induced osteoclastogenesis and/or by alleviating the suppressive action of cancer cells on osteoblastogenesis. Our data revealed that GV does indeed prevent the suppressed osteoblastogenesis and stimulated osteoclastogenesis caused by coculture with MDA-MB-231 cells. Notably, GV was also found to directly suppress MDA-MB-231 proliferation by a mechanism involving apoptotic cell death. These data provide a proof of concept for future in vivo studies to examine the potential of GV to suppress breast cancer growth in vivo and to alleviate skeletal impairments due to cancer cells.

\section{Materials and methods}

Materials. Dulbecco's modified Eagle's medium (DMEM) with $4.5 \mathrm{~g} / 1$ glucose, L-glutamine and sodium pyruvate and antibiotics (penicillin and streptomycin) were purchased from Corning Cellgro (Mediatech, Inc. Manassas, VA, USA). $\alpha$-minimum essential medium ( $\alpha$-MEM) was purchased from Sigma-Aldrich (St. Louis, MO, USA). Fetal bovine serum (FBS) was purchased from HyClone (Logan, UT, USA). Alizarin red, lipopolysacharide (LPS), caspase-3 inhibitor and all other reagents were purchased from Sigma-Aldrich unless otherwise specified.

Cancer cells. The human breast cancer cell line MDA-MB-231 and human pancreatic cancer cell line MIA PaCa-2 were obtained from the American Type Culture Collection (Manassas, VA, USA). MDA-MB-231 cells were derived from a pleural effusion of mammary gland/breast metastases and lacked estrogen, progesterone and human epithelial growth factor type 2 (HER2) receptors and were therefore considered as triple-negative (19).

Proliferation of the cancer cells. Breast cancer MDA-MB231 or MIA PaCa- 2 cells $\left(1 \times 10^{5} / \mathrm{ml} /\right.$ well $)$ were cultured in 24-well plates in DMEM containing $10 \% \mathrm{FBS}$ and $1 \% \mathrm{P} / \mathrm{S}$ in the presence or absence of $\mathrm{GV}(1,10,50,100$ or $200 \mathrm{nM})$ for $1,3,7$ or 14 days in a water-saturated atmosphere containing $5 \% \mathrm{CO}_{2}$ and $95 \%$ air at $37^{\circ} \mathrm{C}$. After culture, the cells were detached from culture dishes and quantitated as previously described $(20,21)$.

In separate experiments, MDA-MB-231 cells $\left(1 \times 10^{5}\right.$ cells/ml/well) were cultured using a 24-well plate in DMEM containing $10 \% \mathrm{FBS}$ and $1 \% \mathrm{P} / \mathrm{S}$ in the absence of GV for 7 days until reaching confluency, and then the cells were cultured in the presence of $\mathrm{GV}(1,10,50,100$ or $200 \mathrm{nM})$ with or without caspase-3 inhibitor ( $5 \mu \mathrm{M})$ for 3 days (22). After culturing, the cells were detached from each culture dish and counted.

Cell counting. After trypsinization of each culture dish using $0.2 \%$ Trypsin plus $0.02 \%$ EDTA in $\mathrm{Ca}^{2+} / \mathrm{Mg}^{2+}$-free phosphatebuffered saline (PBS) for $2 \mathrm{~min}$ at $37^{\circ} \mathrm{C}$, detached cells were collected after centrifugation. The cells were resuspended in PBS and stained with eosin. Cell numbers were counted under a microscope using a hemocytometer plate. For each dish, we took the average of the two countings. The cell number was presented as cell number/well.

Animals and bone marrow cells. Female mice (CD1-Elite, wild-type, 2-months old), which were purchased from Charles River, were housed in a pathogen-free facility, and all procedures and protocols were approved by the Institutional Animal Care and Use Committee at Emory University. The femur and tibia tissues were removed immediately after sacrifice. Bone marrow cells were isolated with a procedure of sterilization from the femoral and tibial tissues.

Osteoclastogenesis in bone marrow cell culture. To determine the effects of breast cancer cells on osteoclastogenesis in whole bone marrow, bone marrow cells $\left(2 \times 10^{5}\right.$ cells $/ \mathrm{ml} /$ well $)$ were cultured in DMEM containing $10 \% \mathrm{FBS}$ and $1 \% \mathrm{P} / \mathrm{S}$ in 24-well plates (1.0 ml/well) (18). The cells were cultured with or without LPS (10 $\mu \mathrm{g} / \mathrm{ml}$ of medium) for 3 days in the presence or absence of GV (10, 50, 100 and $200 \mathrm{nM})$. The cells were fed by replacing $50 \%(0.5 \mathrm{ml})$ of old medium with fresh medium containing fresh LPS $(10 \mu \mathrm{g} / \mathrm{ml}$ of medium) 

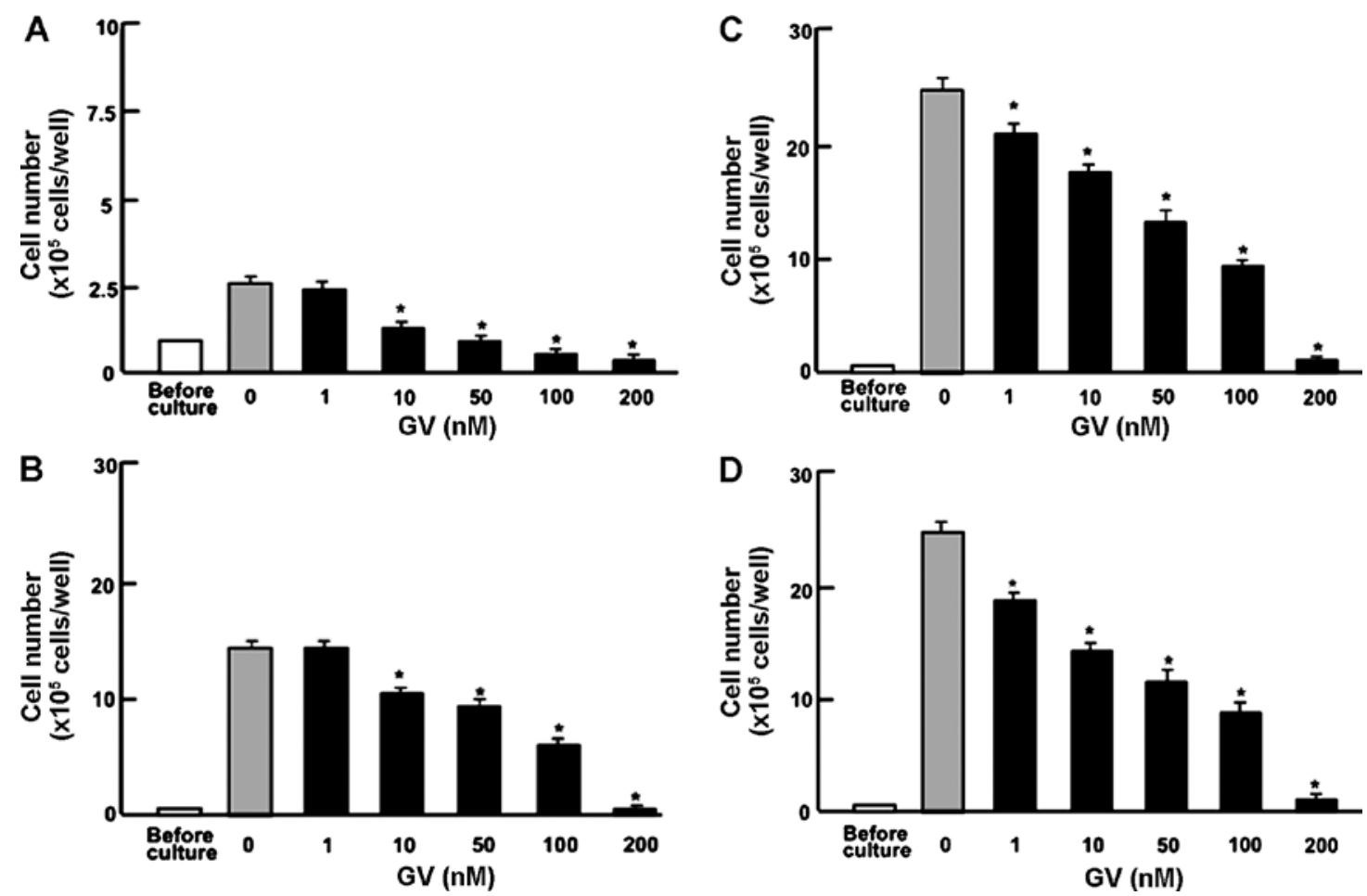

Figure 1. Gentian violet (GV) suppresses the proliferation of human breast cancer MDA-MB-231 cells in vitro. Cells were cultured in DMEM in the presence or absence of GV (1-200 nM) for (A) 1, (B) 3, (C) 7 or (D) 14 days. After culturing, the number of attached cells on the dish was counted. Data are presented as mean \pm SD of 2 independent experiments. " $\mathrm{p}<0.001$ vs. control (grey bar); one-way ANOVA, Tukey-Kramer post hoc test.

and $\mathrm{GV}(10,50,100$ and $200 \mathrm{nM})$ as appropriate. Cultures were maintained for an additional 4 days (18). After 7 days of culture, the cells were washed with PBS and fixed with $10 \%$ neutralized formalin-phosphate $(\mathrm{pH} 7.2)$ for $10 \mathrm{~min}$. The plates were stained with tartrate-resistant acid phosphatase (TRACP), a specific marker of osteoclasts (23) by incubating for $90 \mathrm{~min}$ at room temperature in acetate buffer $(\mathrm{pH} 5.0)$ containing naphthol AS-MX phosphate (Sigma) as a stain for the reaction product, in the presence of $10 \mathrm{mM}$ sodium tartrate. TRACP-positive multinucleated cells (MNCs) containing 3 or more nuclei were counted as osteoclast-like cells. MNCs scored were the mean \pm SD of 6 cultures.

Osteoblast mineralization in cocultures of bone marrow and breast cancer cells. To determine the effects of breast cancer cells on bone marrow osteoblastogenesis and mineralization, we used mineralization medium (MM) containing ascorbic acid $(100 \mathrm{ng} / \mathrm{ml})$ and $4 \mathrm{mM} \beta$-glycerophosphate in DMEM with $10 \% \mathrm{FBS}$ and $1 \% \mathrm{P} / \mathrm{S}$ using 12 -well plates. Bone marrow cells $\left(1 \times 10^{6} \mathrm{cells} / \mathrm{ml} /\right.$ well $)$ were cultured for 3 days at $37^{\circ} \mathrm{C}$ in a humidified $5 \% \mathrm{CO}_{2}$ atmosphere, and then cocultured with breast cancer MDA-MB-231 cells $\left(1 \times 10^{4}\right.$ cells/well $)$ in the presence or absence of $\alpha$-MEM and MM with either vehicle or GV (1, 10 or $100 \mathrm{nM})$ for 18 days (18). Medium was changed every 3 days. After culturing, the cells were washed with PBS and stained with Alizarin red S stain. For quantification, 10\% cetylpyridinium chloride solution was added to each well to elute the dye (18). After complete elution, the absorbance was measured at $570 \mathrm{~nm}$ on a microtiter plate reader.

Coculture of preosteoblastic MC3T3 cells with breast cancer cells. Preosteoblastic MC3T3 cells $\left(2 \times 10^{5}\right.$ cells/ $\left.1 \mathrm{ml} / \mathrm{well}\right)$ were cultured using a 12 -well plare in $\alpha$-MEM containing $10 \% \mathrm{FBS}$ and $1 \% \mathrm{P} / \mathrm{S}$, and 3 days later the culture medium was replaced with DMEM (containing 10\% FBS and 1\% $\mathrm{P} / \mathrm{S}$ ) in the presence or absence of MM containing ascorbic acid $(100 \mathrm{ng} / \mathrm{ml})$ and $4 \mathrm{mM} \beta$-glycerophosphate. After 3 days, the osteoblastic cells were cocultured with the addition of breast cancer MDA-MB-231 bone metastatic cells $\left(1 \times 10^{3}\right.$ or $1 \times 10^{4}$ cells $/ \mathrm{ml} /$ well) in $\alpha$-MEM containing MM in the presence or absence of GV (1, 10 and $100 \mathrm{nM})$ for 18 days (18). Medium was changed every 3 days. After culturing, the cells were washed with PBS and stained with Alizarin red S stain. For quantification of calcium deposition, after washing of cells to remove unbound dye, Alizarin red $\mathrm{S}$ was eluted with $10 \%$ cetylpyridinium chloride and the absorbance was read at $570 \mathrm{~nm}$ on a microtiter plate reader.

Statistical analysis. Statistical significance was determined using GraphPad InStat version 3 for Windows XP (GraphPad Software, Inc., La Jolla, CA, USA). Multiple comparisons were performed by one-way analysis of variance (ANOVA) with Tukey-Kramer multiple comparisons post-test for parametric data as indicated. $\mathrm{p}<0.05$ was considered to indicate a statistically significant result.

\section{Results}

$G V$ suppresses proliferation of $M D A-M B-231$ and $M I A$ $\mathrm{PaCa}-2$ cells. To determine the effects of GV on the proliferation of human breast cancer cells, MDA-MB-231 cells were cultured in the presence of GV for 1-14 days. Addition of GV (1-200 nM) led to a significant dose dependent suppression of cell proliferation by day 1 (Fig. 1A), day 3 (Fig. 1B), 

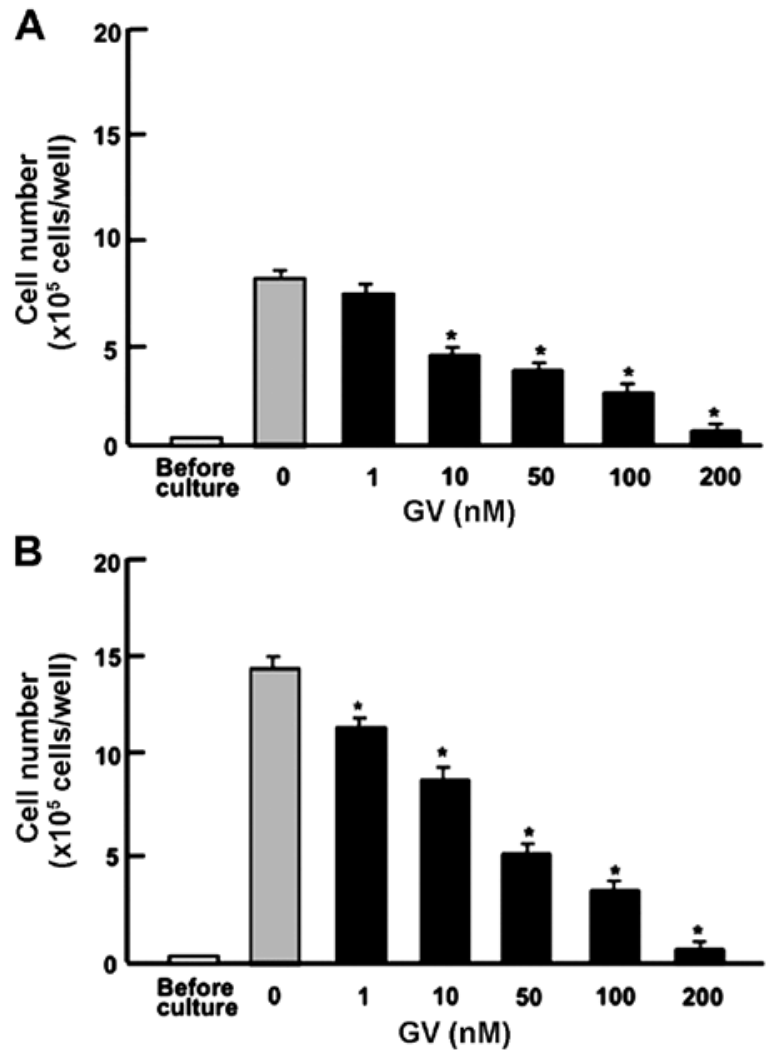

Figure 2. Effect of gentian violet (GV) on the proliferation of human pancreatic cancer MIA PaCa-2 cells in vitro. Cells were cultured in the presence of GV $(1,10,50,100$ or $200 \mathrm{nM})$ for (A) 3 or (B) 7 days. After culturing, the number of attached cells on the dish was counted. Data are presented as mean \pm SD of 2 independent experiments. " $p<0.001$ vs. control (grey bar); one-way ANOVA, Tukey-Kramer post hoc test.

day 7 (Fig. 1C) and day 14 (Fig. 1D). To investigate whether these effects are unique to breast cancer cells, we further examined the effects of GV on the cell proliferation of MIA PaCa-2 human pancreatic cancer cells. As with MDA-MB231 cells, proliferation was suppressed by GV (1-200 nM) at day 3 (Fig. 2A) and day 7 (Fig. 2B). Taken together, these data reveal for the first time the antiproliferative effects of $\mathrm{GV}$ on human breast and pancreatic cancer cells in vitro.

$G V$ stimulates apoptotic cell death of MDA-MB-231 breast cancer cells. We previously reported that GV promotes osteoblast differentiation at doses of up to $100 \mathrm{nM}$ in vitro. Even though GV suppressed osteoclastic differentiation of the monocytic (osteoclast precursor) cell line RAW264.7, $\mathrm{GV}$ did not promote apoptosis at the same bioactive doses (10-100 nM) (17). To investigate whether the suppression of proliferation by GV on MDA-MB-231 cells involves apoptosis or another mechanism, confluent MDA-MB-231 cells were treated with $\mathrm{GV}(10,50$ and $100 \mathrm{nM})$ with or without caspase-3 inhibitor $(5 \mu \mathrm{M})$ for an additional 3 days (Fig. 3A and B). The data revealed that in the presence of the caspase- 3 inhibitor, MDA-MB-231 cells were completely protected from the inhibitory effects of GV. These data demonstrated that unlike the effect of GV on osteoblast and osteoclast precursor cell lines, MDA-MB-231 breast cancer cells did undergo apoptosis in the presence of low-dose GV.
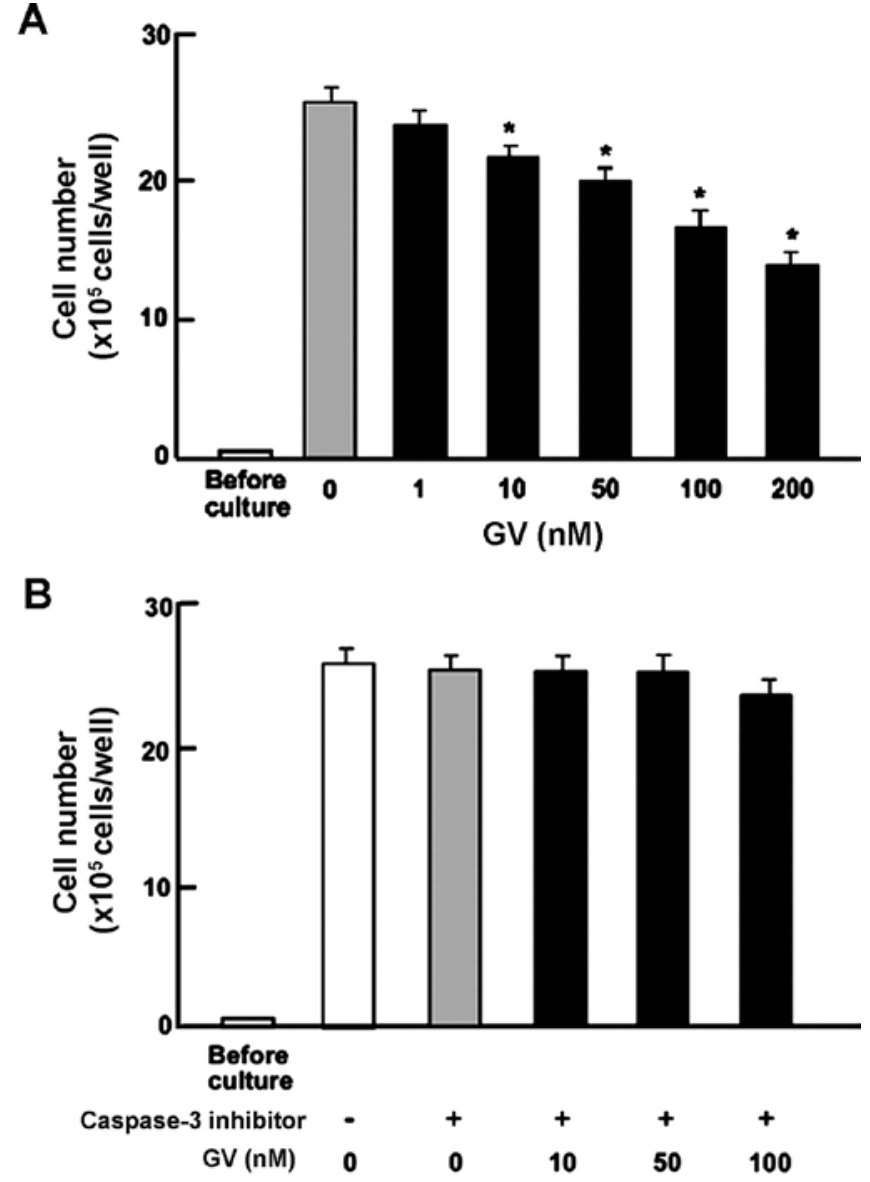

Figure 3. Gentian violet (GV) stimulates apoptotic cell death in human breast cancer MDA-MB-231 cells in vitro. (A) Cells were cultured for 7 days until reaching confluency, and then the cells were cultured for an additional 3 days in the presence of $\mathrm{GV}(1,10,50,100$ or $200 \mathrm{nM})$. (B) Cells were cultured for 7 days until reaching confluency, and then the cells were cultured for an additional 3 days in the presence of $\mathrm{GV}(10,50$ or $100 \mathrm{nM})$ with or without caspase-3 inhibitor $(5 \mu \mathrm{M})$. After culturing, the number of attached cells on the dish was counted. Data are presented as mean $\pm \mathrm{SD}$ of 2 independent experiments. " $\mathrm{p}<0.001$ relative to control (grey bar); one-way ANOVA, Tukey-Kramer post hoc test.

$G V$ inhibits the osteoclast formation in bone marrow cultures in vitro. We previously reported that GV blocks the RANKLinduced differentiation of RAW264.7 cells into mature osteoclasts (17). Although RANKL is a key osteoclastic cytokine, activation of Toll-like receptors on osteoblasts and monocytes by the bacterial-derived protein lipopolysaccharide (LPS), leads to production of inflammatory cytokines such as IL-1 and TNF- $\alpha$ from monocytes and by RANKL production from osteoblasts causing significant osteoclastogenesis (13). To test the ability of GV to moderate osteoclastogenesis in a more physiological setting we used a primary bone marrow culture system in which whole bone marrow was treated with LPS in vitro with or without GV (50-200 nM) for 7 days (Fig. 4A). LPS caused a marked increase in osteoclastogenesis in the bone marrow cells in vitro. This increase was dose-dependently prevented by addition of GV (10-200 nM). These data confirm the capacity of GV to suppress the differentiation of osteoclasts in bone marrow and further show for the first time that GV blocks osteoclastogenesis in a primary bone marrow culture system. 
A

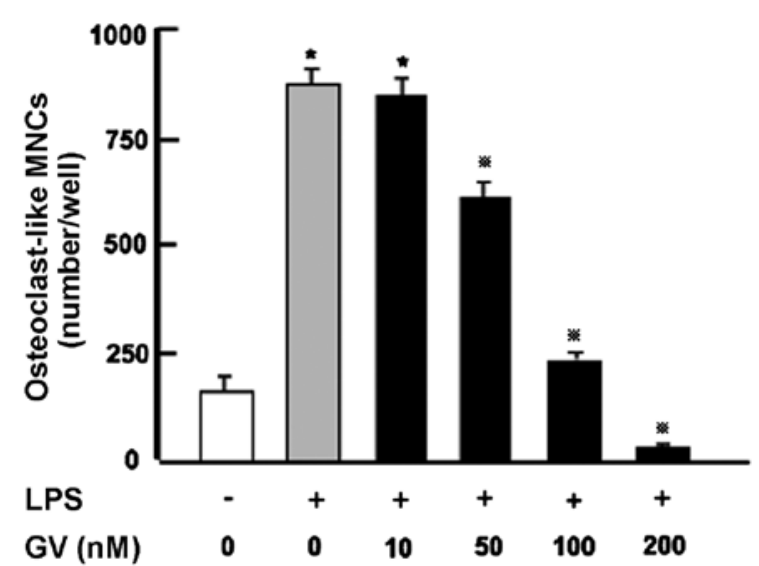

B

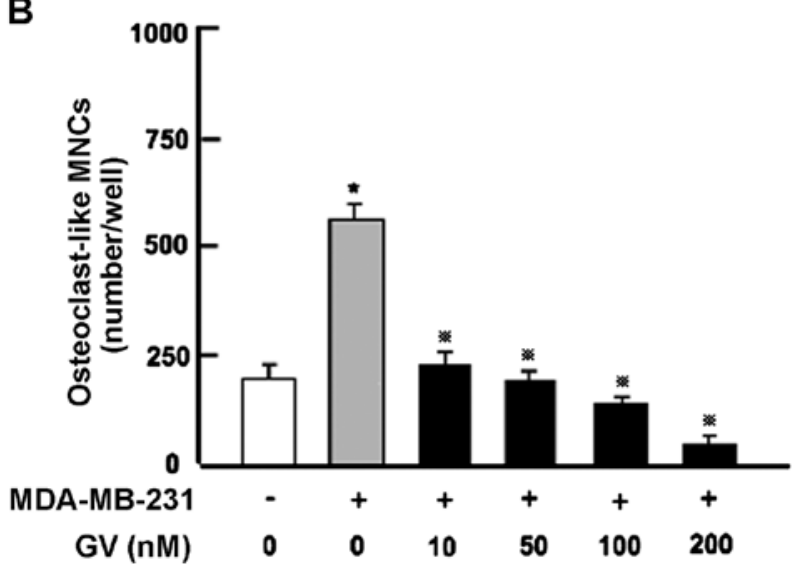

Figure 4. Gentian violet (GV) suppresses osteoclastogenesis enhanced by coculture with mouse bone marrow and human breast cancer MDA-MB-231 cells in vitro. (A) Bone marrow cells were cultured in the presence of LPS $(1 \mu \mathrm{g} / \mathrm{ml})$ with or without $\mathrm{GV}(10,50,100$ or $200 \mathrm{nM})$ for 7 days. (B) Bone marrow cells were cultured in the presence or absence of GV $(10,50,100$ or $200 \mathrm{nM}$ ) and/or addition of MDA-MB-231 cells without LPS for 7 days. After culture, TRAP was used for staining. TRAP ${ }^{+}$multinucleated cells ( 3 or more nuclei) were quantitated and averaged for 8 independent wells for each data point. Data are presented as mean \pm SD of 2 independent experiments. " $\mathrm{p}<0.001$ relative to control (white bar) or " $\mathrm{p}<0.001$ vs. control (grey bar); one-way ANOVA, Tukey-Kramer post hoc test.

$G V$ alleviates the pro-osteoclastogenic activity of MDA-MB231 cells in vitro. Since GV blocks both osteoclastogenesis and MDA-MB-231 breast cancer cell proliferation, we next investigated whether GV alleviates the osteoclast formation promoted by human breast cancer. To this end, MDA-MB-231 cells were cocultured with mouse bone marrow cells in vitro as previously described (18). Osteoclastogenesis was markedly enhanced by coculture with MDA-MB-231 cells (Fig. 4B) and was significantly suppressed by addition of GV (10-200 nM).

$G V$ alleviates the anti-osteoblastogenic activity of $M D A-M B-231$ cells in vitro. Next, we examined whether GV alleviates the suppressive effect of MDA-MB-231 cells on osteoblast differentiation and mineralization activity using the MC3T3 preosteoblastic cell line (Fig. 5A) as well as primary bone marrow cultures (Fig. 5B). After 3 days of culture, preosteoblastic MC3T3 cells were cocultured with MDA-MB-231 cells in MM in the presence or absence of GV
A
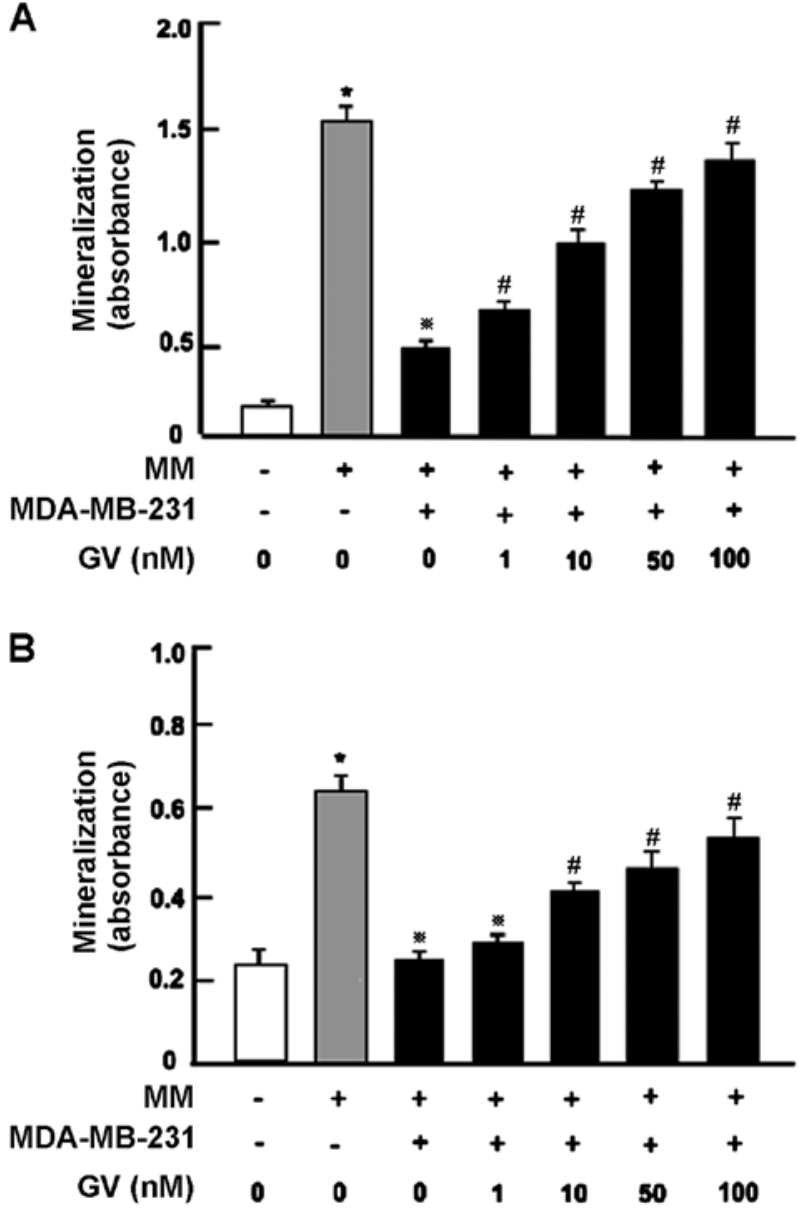

Figure 5. Gentian violet $(\mathrm{GV})$ prevents the suppression of mineralization in (A) bone marrow and (B) preosteoblastic MC3T3 cells induced by coculture with human breast cancer MDA-MB-231 cells in vitro. (A) Bone marrow cells were cultured in DMEM in the presence or absence of mineralization medium (MM). After 3 days, the cells were cocultured with the addition of MDA-MB-231 cells in DMEM containing MM in the presence or absence of $\mathrm{GV}(1,10$ or $100 \mathrm{nM}$ ) for 18 days. (B) Osteoblastic cells were cultured in the presence or absence of MM for 3 days, and then the cells were cocultured with the addition of MDA-MB-231 cells in medium containing MM in the presence or absence of $\mathrm{GV}(1,10$ or $100 \mathrm{nM})$ for an additional 18 days. After culturing, the cells were stained with Alizarin red S stain. Data are presented as mean \pm SD of 2 independent experiments. ${ }^{*} \mathrm{p}<0.001$ relative to the control without MM (white bar) or " $\mathrm{p}<0.001$ vs. the control with MM (grey bar). ${ }^{\#} \mathrm{p}<0.001$ vs. MM without GV (black bar); one-way ANOVA, Tukey-Kramer post hoc test.

(1, 10 and $100 \mathrm{nM}$ ) for an additional 18 days (Fig. 5A). We previously reported (18), that coculture with MDA-MB-231 cells potently suppressed mineralization in osteoblastic cells. Importantly, this suppression was significantly prevented by GV (1-100 nM) (Fig. 5A).

Finally, we cocultured primary bone marrow cells containing osteoblast precursors (Fig. 5B) with MDA-MB-231 cells in vitro. Mouse bone marrow cells were cultured in the presence or absence of MM. After 2 days, the cells were cocultured with the addition of MDA-MB-231 cells in the presence or absence of $\mathrm{GV}(1,10$ or $100 \mathrm{nM})$ for 18 days which led to mineralization. Mineralization in the bone marrow cells was potently suppressed by coculture with the MDA-MB-231 cells. This suppression was prevented by GV (1-100 nM) (Fig. 5B). 


\section{Discussion}

The central finding of the present study is that GV mediates a suppressive effect on proliferation, and a stimulatory effect on apoptotic cell death, in human breast cancer MDA-MB-231 cells in vitro. Moreover, both the suppressed osteoblastogenesis and enhanced osteoclastogenesis induced by coculture with MDA-MB-231 cells were prevented in the presence of $\mathrm{GV}$ in vitro. Thus, GV has significant potential to alleviate the skeletal abnormalities and metastatic engraftment of human breast cancer cells in vivo.

We previously reported that GV stimulates osteoblastic mineralization and suppresses RANKL-induced osteoclastogenesis using preosteoblastic MC3T3 cells and RAW267.4 osteoclast precursors in vitro (17). Due to the limitations of the cell lines we further demonstrated the capacity of GV to mediate these responses in the context of whole bone marrow systems ex vivo. These models represent a more physiological response as would occur in vivo in the context of whole bone marrow. In vivo, RANKL is supplied by bone marrow cells such as osteoblasts. Consequently, rather than providing exogenous RANKL as in our prior studies we stimulated RANKL production from bone marrow cells using LPS. As previously reported for the cell lines, GV potently suppressed osteoclastogenesis and enhanced osteoblast mineralization in whole bone marrow. These studies provide a proof of concept for future studies of GV action in intact animals in vivo.

We previously reported that these differential actions of $\mathrm{GV}$ on osteoclast and osteoblasts stem from the capacity of $\mathrm{GV}$ to suppress activation of the NF- $\kappa \mathrm{B}$ signal transduction pathway that is required for osteoclastogenesis, yet inhibitory to osteoblast differentiation and activity (17). We speculated that $\mathrm{GV}$ is an inhibitor of NF- $\kappa \mathrm{B}$ activation and holds promise for modulation of bone turnover to promote a balance between bone formation and bone resorption, favorable to the gain of bone mass. In the context of breast cancer, one action of GV may thus be to restore bone turnover, deregulated by cancer cells. Breast cancer cells are known to produce RANKL, which plays a pivotal role in formation from preosteoclastic cells to mature osteoclasts (7-13). Indeed the capacity of MDA-MB-231 cells to promote osteoclastogenesis in our bone marrow culture system may be due to RANKL production.

As reported for in vivo studies of breast cancer cells in mice $(18,24)$ and in vitro using MC3T3 cell lines (17), osteoblastic mineralization in mouse bone marrow cells was markedly suppressed after coculture with breast cancer MDA-MB-231 cells in vitro. The mechanisms driving osteoblast suppression are not presently clear, yet could be related to production of TNF- $\alpha$, a cytokine that is known to be produced by breast cancer cells $(10,11)$. TNF- $\alpha$ suppresses osteoblastic mineralization by activation of $\mathrm{NF}-\kappa \mathrm{B}$ signaling $(17,25)$. Owing to the anti-NF- $\mathrm{BB}$ activity of GV previously reported by us (17), we speculate that the capacity of GV to relieve the inhibitory effects of MDA-MB-231 breast cancer cells on osteoblastic mineralization relates to suppression of TNF- $\alpha$ induced NF- $\kappa \mathrm{B}$ signaling in osteoblasts, the direct stimulation of osteoblastic mineralization, the induction of apoptosis in MDA-MB-231 cells or a combination of these mechanisms. Whether the actions of GV were mediated through actions on bone cells or on cancer cells, or on both, remains to be determined. Nonetheless, irrespective of the site of action, the data suggest that $\mathrm{GV}$ is a useful agent to break the vicious cycle, diminish growth and metastases of breast cancer cells, and repair preexisting damage to the skeleton caused by bone metastases. GV restores a favorable balance between bone formation and resorption to negate the damaging effects of cancer metastases. Future studies in vivo are needed to establish the therapeutic potential of GV.

In conclusion, the present study demonstrated that GV suppressed proliferation and stimulated apoptotic cell death in human breast cancer MDA-MB-231 cells in vitro, and that it alleviated the suppression of osteoblastogenesis and stimulated the osteoclastogenesis induced by coculture with MDA-MB-231 cells in vitro. Thus, GV may be a new and useful therapeutic tool for breast cancer bone metastasis.

\section{References}

1. Raggatt LJ and Partridge NC: Cellular and molecular mechanisms of bone remodeling. J Biol Chem 285: 25103-25108, 2010.

2. Weitzmann MN and Pacifici R: Estrogen deficiency and bone loss: An inflammatory tale. J Clin Invest 116: 1186-1194, 2006

3. Johnell $\mathrm{O}$ and Kanis JA: An estimate of the worldwide prevalence and disability associated with osteoporotic fractures. Osteoporos Int 17: 1726-1733, 2006

4. Boyce BF, Yoneda T and Guise TA: Factors regulating the growth of metastatic cancer in bone. Endocr Relat Cancer 6: 333-347, 1999.

5. Mundy GR: Metastasis to bone: Causes, consequences and therapeutic opportunities. Nat Rev Cancer 2: 584-593, 2002.

6. Roodman GD: Mechanism of bone metastasis. N Engl J Med 350: 1655-1664, 2004

7. Akhtari M, Mansuri J, Newman KA, Guise TM and Seth P: Biology of breast cancer bone metastasis. Cancer Biol Ther 7: 3-9, 2008.

8. Coleman RE: Metastatic bone disease: Clinical features, pathophysiology and treatment strategies. Cancer Treat Rev 27: 165-176, 2001.

9. Chen YC, Sosnoski DM and Mastro AM: Breast cancer metastasis to the bone: Mechanisms of bone loss. Breast Cancer Res 12: 215, 2010.

10. Park BK, Zhang H, Zeng Q, Dai J, Keller ET, Giordano T, Gu K, Shah V, Pei L, Zarbo RJ, McCauley L, Shi S, Chen S and Wang CY: NF-kappaB in breast cancer cells promotes osteolytic bone metastasis by inducing osteoclastogenesis via GM-CSF. Nat Med 13: 62-69, 2007.

11. Gonzalez-Suarez E, Jacob AP, Jones J, Miller R, RoudierMeyer MP, Erwert R, Pinkas J, Branstetter D and Dougall WC: RANK ligand mediates progestin-induced mammary epithelial proliferation and carcinogenesis. Nature 468: 103-107, 2010.

12. Zaidi M, Blair HC, Moonga BS, Abe E and Huang CL: Osteoclastogenesis, bone resorption, and osteoclast-based therapeutics. J Bone Miner Res 18: 599-609, 2003.

13. Weilbaecher KN, Guise TA and McCauley LK: Cancer to bone: a fatal attraction. Nat Rev Cancer 11: 411-425, 2011.

14. Berrios RL and Arbiser JL: Effectiveness of gentian violet and similar products commonly used to treat pyodermas. Dermatol Clin 2011: 69-73, 2011.

15. Perry BN, Govindarajan B, Bhandarkar SS, Knaus UG, Valo M, Sturk C, Carrillo CO, Sohn A, Cerimele F, Dumont D, et al: Pharmacologic blockade of angiopoietin-2 is efficacious against model hemangiomas in mice. J Invest Dermatol 126: 2316-2322, 2006.

16. Zhang X, Zheng Y, Fried LE, Du Y, Montano SJ, Sohn A, Lefkove B, Holmgren L, Arbiser JL, Holmgren A, et al: Disruption of the mitochondrial thioredoxin system as a cell death mechanism of cationic triphenylmethanes. Free Radic Biol Med 50: 811-820, 2011.

17. Yamaguchi M, Vikulina T, Arbiser JL and Weitzmann MN: Suppression of $\mathrm{NF}-\kappa \mathrm{B}$ activation by gentian violet promotes osteoblastogenesis and suppresses osteoclastogenesis. Curr Mol Med 14: 783-792, 2014. 
18. Yamaguchi M, Zhu S, Weitzmann MN, Snyder JP and Shoji M Curcumin analog UBS109 prevents bone marrow osteoblastogenesis and osteoclastogenesis disordered by coculture with breast cancer MDA-MB-231 bone metastatic cells in vitro. Mol Cell Biochem 401: 1-10, 2015.

19. Yoneda T, Williams PJ, Hiraga T, Niewolna M and Nishimura R A bone-seeking clone exhibits different biological properties from the MDA-MB-231 parental human breast cancer cells and a brain-seeking clone in vivo and in vitro. J Bone Miner Res 16: 1486-1495, 2001.

20. Misawa H, Inagaki S and Yamaguchi M: Suppression of cell proliferation and deoxyribonucleic acid synthesis in the cloned rat hepatoma H4-II-E cells overexpressing regucalcin. J Cell Biochem 84: 143-149, 2001.

21. Yamaguchi M and Daimon Y: Overexpression of regucalcin suppresses cell proliferation in cloned rat hepatoma H4-II-E cells: involvement of intracellular signaling factors and cell cycle-related genes. J Cell Biochem 95: 1169-1177, 2005.
22. Izumi $\mathrm{T}$ and Yamaguchi M: Overexpression of regucalcin suppresses cell death in cloned rat hepatoma H4-II-E cells induced by tumor necrosis factor-alpha or thapsigargin. J Cell Biochem 92: 296-306, 2004.

23. Minkin C: Bone acid phosphatase: Tartrate-resistant acid phosphatase as a marker osteoclast function. Calcif Tissue Int 34: 285-290, 1982.

24. Yamaguchi M, Zhu S, Zhang S, Wu D, Moore TM, Snyder JP and Shoji M: Curcumin analogue UBS109 prevents bone loss in breast cancer bone metastasis mouse model: Involvement in osteoblastogenesis and osteoclastogenesis. Cell Tissue Res 357: 245-252, 2014

25. Li Y, Li A, Strait K, Zhang H, Nanes MS and Weitzmann MN: Endogenous TNFalpha lowers maximum peak bone mass and inhibits osteoblastic Smad activation through NF-kappaB. J Bone Miner Res 22: 646-655, 2007. 